\title{
Developments in Techniques and Algorithms for Materials-based Electron Tomography
}

\author{
P.A. Midgley*. J.R. Tong***, I. Arslan*** and J.-C. Hernandez* \\ * Department of Materials Science and Metallurgy, University of Cambridge, Pembroke Street, \\ Cambridge, CB2 3QZ, UK \\ ** Department of Electrical and Electronic Engineering, University of Bath, Bath, BA2 7AY, UK \\ *** Department of Chemical Engineering and Materials Science, University of California-Davis, \\ One Shields Ave, Davis, CA 95616
}

There has been much progress made in the past decade to develop new imaging modes, techniques and reconstruction algorithms for electron tomography as practiced in materials science. Many of these have come about because of the difference in image contrast in micrographs of materials specimens (crystallographic contrast, strong absorption and high angle scattering etc) compared to those in the life sciences. In addition, the ability of most materials specimens to retain their structure when bombarded by electrons has enabled long acquisition modes such as EFTEM to be used for tomography. Concurrent with new imaging modes has been the further development of acquisition schemes, such as dual axis tomography and the use of needle specimens, which can work especially well for materials specimens.

In dual axis tomography, two tilt series of images are acquired about axes which are (conventionally) mutually perpendicular. This benefits of such acquisition is that more overall information is collected about the specimen and artefacts that arise from the missing wedge can be minimized for a given tilt range, see Fig. 1(a). Dual axis acquisition can now be married with iterative reconstructions. In one implementation of this, called ADA-SIRT [1], the iteration alternates between the two data sets to constrain the overall reconstruction to best fit both sets simultaneously. Conventional dual axis acquisition combines two independently reconstructed data sets but of course the data contained in the two sets are not independent and come from the same single object. An example of an ADA-SIRT reconstruction is shown in Fig. 1(b).

One of the major issues with dual-axis tomography however is the need to take into account any geometric distortions that occur via scan distortions (for STEM) or pin-cushion distortions (for TEM). In addition there will always be a finite amount of information missing. To address this problem there is now a drive in the community towards using needle specimens which can be rotated completely around the tilt axis [2]. No missing wedge results and thus reconstructions should be free from associated artefacts, enabling true nano-metrology. An example of a needle-specimen reconstruction is shown in Figure 2(a).

Alternatively, artefacts can be minimized by applying further constraints on the reconstruction. Iterative procedures constrain the reconstruction to best fit the available data (normally the images in the tilt series) but in principle the reconstruction can be further constrained if other a priori information is known. Such information may be about the nature of the object, for example how many densities (grey levels in each reconstructed slice) are present. Often this information is known: for example if examining elemental nanoparticles on a carbon support then three 'densities' are expected (nanoparticle, carbon, vacuum). This information can be used to strongly constrain the 
reconstruction yielding fewer reconstruction artefacts from limited data sets, as an example see Figure 2(b). The discrete nature of the object lends itself to applying 'discrete tomography' algorithms, such as DART [3,4]. At very high resolution it is hoped that the discrete (atomic) nature of any object can be used in a similar way to improve 3D reconstructions of atomic structures [5].

References

[1] Tong J.R., Arslan I. and Midgley P.A. J. Structural Biology 153 (2006) 55.

[2] Kawase N., Kato M., Nishioka H. and Jinnai H. Ultramicroscopy 107 (2007) 8.

[3] Batenburg K.J. and Sijbers J. Proc. ICIP IV (2007) 133.

[4] Batenburg K.J. et al. Ultramicroscopy (2009) in press.

[5] This research was supported by the Royal Society, the EPSRC and by the European Union Framework 6 program under a contract for an Integrated Infrastructure Initiative (Reference 026019 ESTEEM). Dr K.J. Batenburg is thanked for providing Figure 2(b). The authors thank P. Alivisatos, E. Coronado and M. Galtrey for providing specimens.

(a)

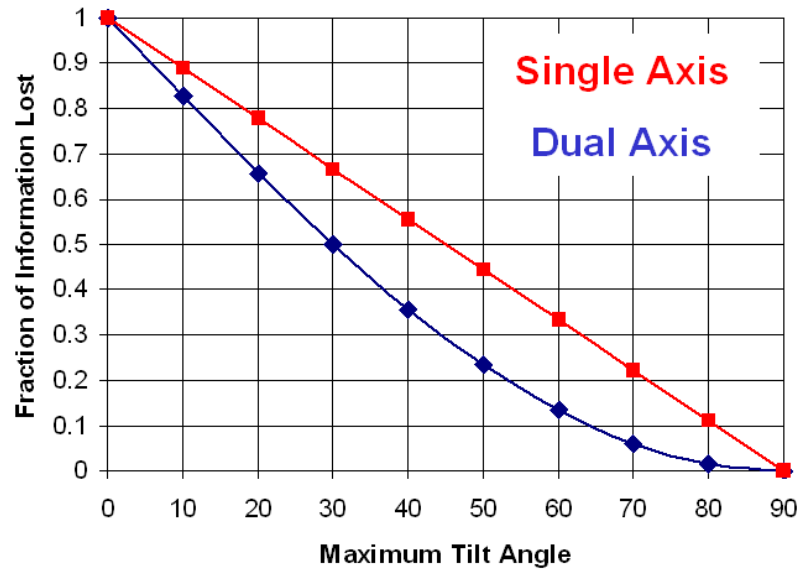

(b)

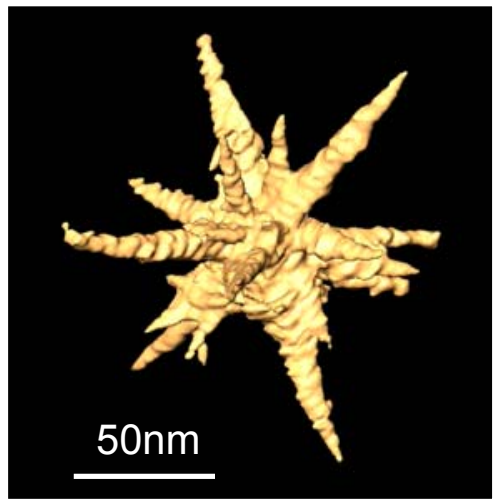

FIG. 1. (a) A comparison of the information lost using limited tilt angles in single and dual axis series. (b) An example of an iterative reconstruction from dual axis data showing a CdTe 'multipod'.
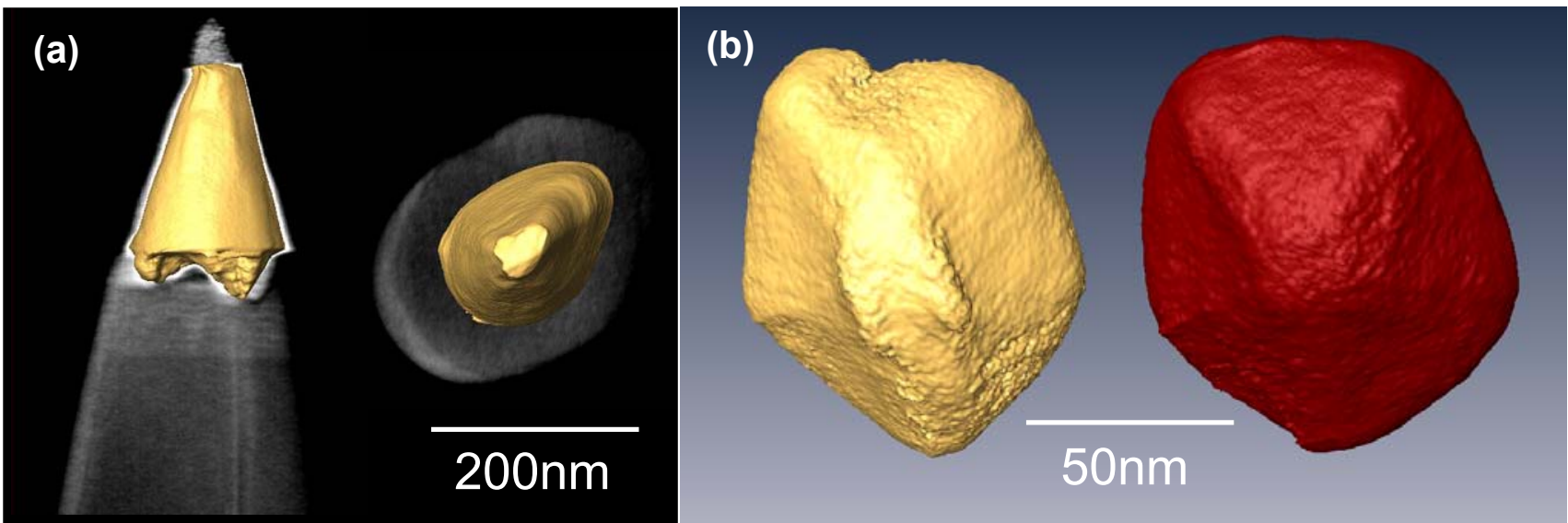

FIG. 2. (a) A side view (left) and top view (right) of a needle specimen of an InGaN/GaN device showing a dislocation image running from a V-pit. (b) A comparison of a SIRT-reconstructed (left) and DART-reconstructed (right) gold nanoparticle (adapted from [4]); only 15 images were used for the reconstruction. 